\title{
THE COMBINATION OF POLAR MOTION OBSERVATIONS
}

\author{
Clark R. Wilson \\ Center for Space Research \\ Department of Geological Sciences \\ The University of Texas at Austin
}

\author{
R. 0. Vicente \\ Faculty of Sciences \\ Department of Applied Mathematics \\ Lisbon, Portuga1
}

\begin{abstract}
Polar motion data for the period 1981-1985 are used to obtain a combined solution from Doppler, Satellite Laser Ranging, and Astrometric observations. The combined solution is a weighted average of the three series, with weights determined from reported errors which are scaled so that they agree with errors estimated from differences among the various series. The combined solution is effective in removing spurious deviations in the pole path which appear in a single series. However, we also show that estimated errors can be unreliable when derived from short time series, when one series is much less noisy than the others. Thus, a combined solution where weights depend upon estimated errors can yield poor results, and we demonstrate this effect by comparing a combined solution for 198485 with the independent IRIS series.
\end{abstract}

\section{INTRODUCTION}

The new methods of observing polar motion (Doppler, 1aser ranging to satellites, and VLBI) have produced a number of estimates of the pole coordinates based on quite different reduction techniques. It is important to compare these results among themselves and with the estimates obtained by classical methods which have been practiced for the past nine decades. Furthermore, it is useful to combine the various independent observations, since a combined solution can be superior to the best individual series if it is properly obtained.

The most straightforward combination methods are linear and use a simple weighted average of the available data to obtain the combined solution. (Feisse1, 1982; Vicente and Wilson, 1986). Additiona11y, the combined solution may include some dependence on the past estimate, and Kalman filter theory can be used to determine the best least squares combination of new data and the past estimate (Steppe et al, 1985; Eubanks et a1, 1985; Wilson and Vicente, 1986) 
In the next section we summarize the calculations required for a combined solution. The critical factor is the determination of the relative noise level in the various data sets, and we propose a hybrid estimate based on both reported errors and estimated errors determined from differences among the various series. Polar motion is denoted by the complex variable $M_{t}$, where $t$ denotes time, and the datum for the $k$ th series at time $t$ is the complex scalar $D_{k t}$. The real component of the complex pole coordinate is associated with the $X$ coordinate, and the imaginary component with the negative $Y$ coordinate. When separate standard errors are given for $X$ and $Y$ coordinates, the square root of the sum of their squares is used as the standard error of the complex pole coordinate.

\section{LEAST SQUARES LINEAR COMBINATION METHODS}

If the kth polar motion series gives the pole position at time $t$ as $D_{k t}$, then the unbiased least squares optimum estimate of pole position from the combined series is the linear combination

$$
M_{t}=a_{k} D_{k t}
$$

where repeated subscript $k$ denotes summation and the weights $a_{k}$ sum to unity. There are other possible solutions to the linear combination problem, such as the Kalman filter method described by Wilson and Vicente (1986) in which the estimate depends linearly on both $D_{k t}$ and upon the past estimate $M_{t-1}$. However, we shall concentrate here on solutions of the form of equation 1.

The weighting functions are solutions to the linear equations:

$$
\left(\delta_{k k}+\sigma_{k}^{2} / \sigma_{t}^{2} \delta_{j k}\right) a_{k}=1
$$

where $\sigma_{t}{ }^{2}$ is the variance at time t of the unknown pole position and $\sigma_{k}{ }^{2}$ is the noise variance in the kth series. The assumption underlying equation 2 is that the noise is zero mean, uncorrelated among the various series, and is completely described by its variance.

Clearly the noise does not have zero mean, because systematic differences among the various independent determinations of polar motion are well known. We will treat these systematic differences by removing the mean value from all series, combining them in a linear way by equation 1 , and adding back the mean value of one of the series to the combined solution.

As an example, the weighting functions for the case of 3 series which are constrained to sum to unity are

$$
a_{1}=\frac{\sigma_{2}^{2} \sigma_{3}^{2}}{\dot{\sigma}_{2}^{2} \sigma_{3}{ }^{2}+\sigma_{1}^{2} \sigma_{3}^{2}+\sigma_{1}{ }^{2} \dot{\sigma}_{2}^{2}}
$$


with $a_{2}$ and $a_{3}$ obtained by permuting the subscripts in the numerator When the weights are constrained to sum to unity, the value of $\sigma_{t}^{2}$
does not affect the solution, and only the relative sizes of the $\sigma_{k}^{2}$ are important. Thus, the critical problem in creating the combined solution is accurately determining the values of $\sigma_{k}{ }^{2}$ for each series.

There are three logical choices for the values of $\boldsymbol{\sigma}_{k}{ }^{2}$ to be used in equation 1 or 3 . First (Method 1 ) we may take the reported error at each time sample as being an estimate of the true error. This reported error may be smaller than the true error, as in the case of the Doppler series (Feisse1,1982), and, since the reported error changes from time to time, the weights $a_{k}$ do as well.

A second choice (Method 2) is to estimate the error from the data by taking differences among the series and calculating the error variances using the Allan or conventional variance estimator (Feissel, 1982; Vicente and Wilson, 1986), or by computing the cross correlations among the various series (Vicente and Wilson, 1986). Method 2 relies upon the assumption that the noise in each series is statistically stationary. This is certainly not true in some cases where the number of observations used to compute the pole coordinates is variable, or where instrumentation changes have altered the precision of individual observations. A further limitation of Method 2 is that three or more independent series are required.

A third choice (Method 3) for calculating the errors is to combine the first two approaches. Using the difference series, we compute the noise variances which represent an average for the the entire series of $\mathbf{N}$ samples. Then, we scale the reported variance at time $t$ by the ratio of average reported variance to average estimated variance, so that the average over time agrees with the Method 2 estimates. Thus we have taken advantage of the information contained in multiple independent observations of pole position, and we have used the information concerning the non stationarity of the noise that is contained in the reported errors. In the next section we compare the results obtained by each of these three approaches.

\section{COMBINED SOLUTIONS FOR 1981-1985}

Polar motion data for the period 1981-1985 given at 5 day intervals were taken from the BIH annual reports. We have available the data from 3 techniques for 1981-85: Doppler (DOP) (1981-1983 Sate11ite 1970-671; 1984 Satel1ite 82D01; 1985 Sate11ite 77DO2); Astrometric (AST); and Satellite Laser Ranging from the University of Texas Center for Space Research (CSR); and for 1984-85 the VLBI series (IRIS) also is available. Table I summarizes the data, together with the results of calculations discussed below. 
Table I

5 Day Polar Motion Data

$1981-1985$

\begin{tabular}{lrcc} 
Series Name & \multicolumn{2}{c}{$\begin{array}{c}\text { Sigma } \\
\text { reported }\end{array}$} & $\begin{array}{c}\text { weight } \\
\text { (Method 2) }\end{array}$ \\
\hline CSR & 9.6 & 13.8 & 0.58 \\
AST & 14.1 & 22.8 & 0.22 \\
DOP & 4.6 & 23.4 & 0.20
\end{tabular}

Table II

5 Day Polar Motion Data

1984-1985

\begin{tabular}{lrrrr}
\hline Series Name & \multicolumn{2}{c}{ Sigma } & $\begin{array}{c}\text { weight } \\
\text { reported }\end{array}$ & $\begin{array}{c}\text { Sigma } \\
\text { computed }\end{array}$ \\
\hline & 3.1 & 7.5 & 0.71 & 2.8 \\
CSR & 14.7 & 24.5 & 0.07 & 25.6 \\
AST & 4.4 & 13.6 & 0.22 & 15.4 \\
DOP & 3.1 & & & \\
IRIS & & & &
\end{tabular}

* Computed sigma determined from the conventional variance estimate of difference series after subtracting the mean value.

Figure 1 shows the three series that we have used. Figure 2 a shows the combined solution obtained by Method 1, with new weights recomputed at each time step according to the reported errors. Figure $2 \mathrm{~b}$ shows the combined solution by Method 2, with the same relative weight used for the entire 5 year period, as given in Table $I$. Finally, Figure $2 \mathrm{c}$ shows the combined solution by Method 3, where the reported variances have been adjusted to agree on average with the estimated variances, which are given in Table $I$.

The discontinuity in the CSR series that is evident in Figure la, indicated by the arrow, has a reported error which is large due to inadequate observations, and thus does not represent a true deviation of the pole. However, it persists in the combined solution of Figure $2 \mathrm{~b}$ because the same relative weight is applied to the entire series. Allowing the weights to vary in time (Figures $2 a$ or $2 c$ ) yields a 
combined solution which shows no evidence of this discontinuity because it is properly given low weight.
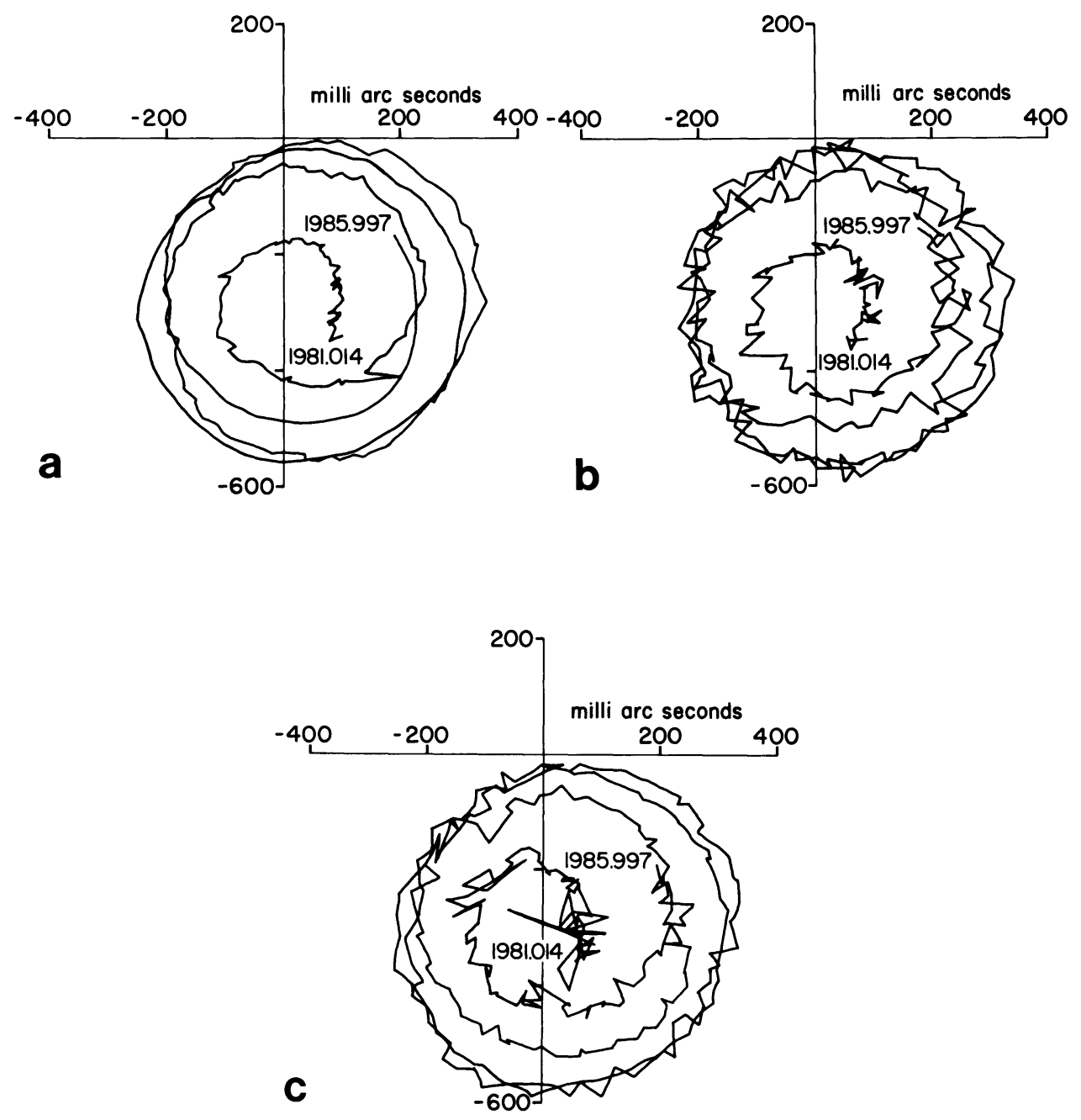

Figure 1. Data for the period 1981-1985. (a) Satelite Laser Ranging; (b) Astrometric; (c) Doppler 


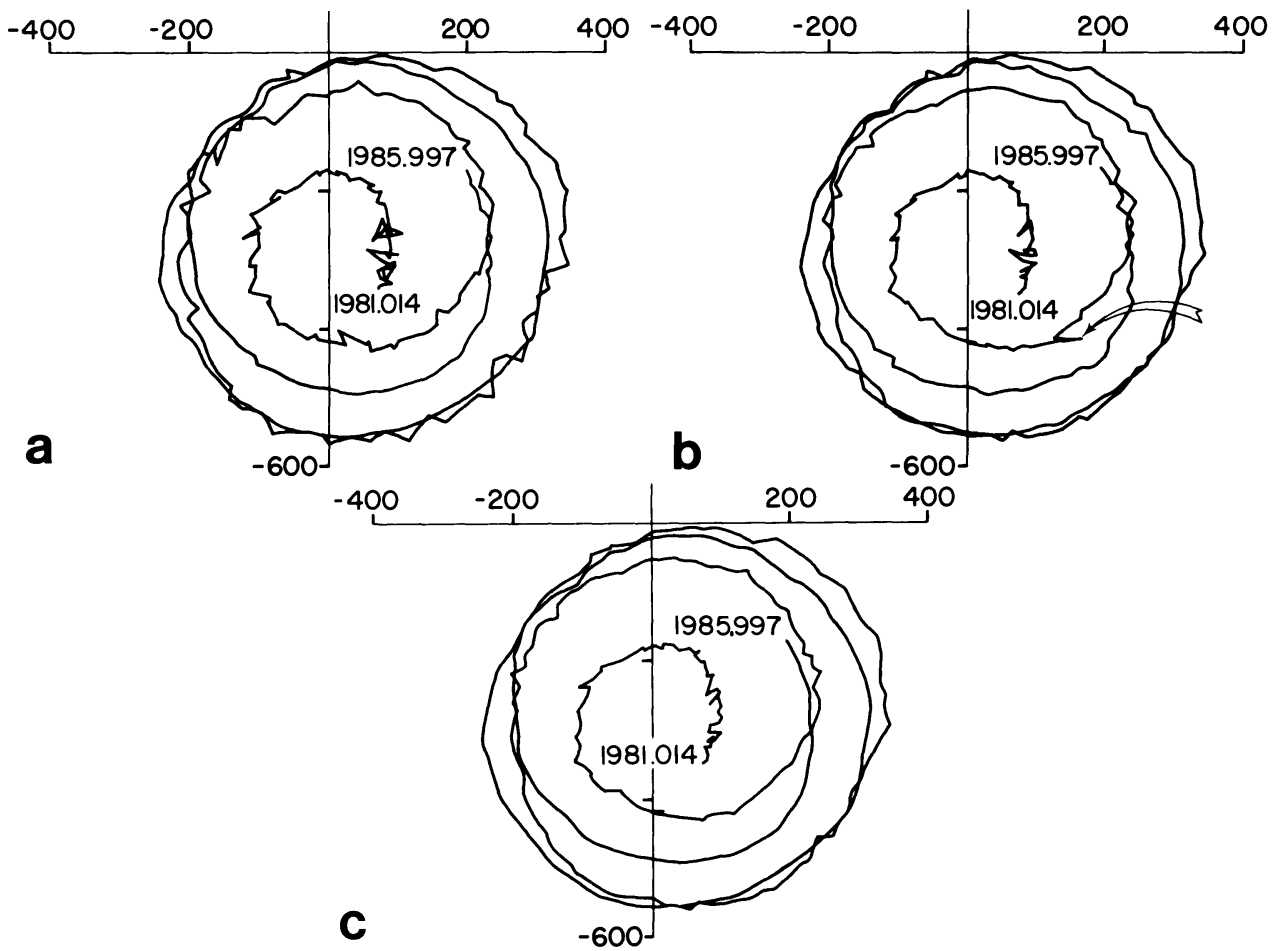

Figure 2. Combined solutions (a) Method 1; (b) Method 2;(c) Method 3.

\section{DISCUSSION}

The general appearance of the the three combined solutions is similar, but the smoothest is obtained by adjusting the reported errors using Method 3, as described in section 2, and illustrated in Figure 2c. This effectively removes the discontinuity that appears in the CSR series, which is associated with a large reported error. The adjustment of the reported errors by the computed errors also reduces the contribution of the DOP series relative to Figure $2 a$, since its reported error is known to be smaller than its true error.

After the beginning of 1984, the IRIS VLBI observations are also available, and it is interesting to compare a combined DOP, AST, and CSR series with the IRIS results. Three combined solutions were determined for 1984-1985 using the Methods 1, 2, and 3. The standard deviations between IRIS, and these 3 combined solutions were 5.6 , 4.5 , and $4.7 \mathrm{mas}$, respectively, after subtracting the mean from each series to remove systematic differences. Table II shows, however that the $\mathrm{rms}$ difference between IRIS and CSR is only $2.8 \mathrm{mas}$, which suggests that CSR alone is better than the combined CSR+DOP+AST series. 
The CSR+AST+DOP combined solution for 1984-1985 is 1ikely to be of poor quality due to erroneous estimates of the error in the three component series. We can identify the source of the problem by assuming that the rms differences between IRIS and the three series CSR, AST, and DOP are good estimates of the true errors. Referring to these values in Table II, we find that the reported error of CSR is approximately correct, while the reported errors for both DOP and AST are much too small. Thus Method 1, based upon reported errors, would fail to produce a good combined solution. The estimated errors for 1984-85 are approximately correct for DOP and AST, but much too large for CSR, so both Methods 2 and 3, which rely on estimated errors, will perform poorly.

The real problem in the combined solution via Method 3 is that the estimated errors for CSR are much too 1arge. The CSR error estimate is poor because the CSR error is much smaller than both AST and DOP series. In order to understand how this occurs, assume that the error variance estimated from differences among the series is a random variable denoted by, $\widehat{\sigma}_{k}{ }^{2}$. The variance of this random variable will tend to be 1 arge for the one series among three which has a low noise leve1. For the case of 3 series ( $k=1$ (CSR), $k=2$ (DOP), and $k=3$ (AST)), the estimate of of error variance in the low noise series ( 1 ) is

$$
\hat{\sigma}_{1}{ }^{2}=.5\left(d_{12}-d_{23}+d_{13}\right)
$$

where the estimated variance of the difference between series 1 and 2 is indicated by $d_{12}$ and so forth. If each of the three estimates $d_{12}, d_{23}, d_{13}$, behaves as a Chi-squared distributed random variable, then its variance will be proportional to its expected value, which will be 1 arge for the estimates involving the two noisy series $(k=2$ and 3). Thus, the estimate of error for the CSR series ( $k=1)$, that has very low noise will tend to be poor because it depends upon quantities like $d_{23}$ which have a large variance.

Thus, a proper combined solution in this case is not obtained with any of the three proposed methods. This leads us to propose a fourth method, which is to use the reported errors for CSR, but to adjust the reported errors by the estimated according to Method 3 for the AST and DOP series. When this is done, the combined solution for 1984-85 gives the majority of weight ( $94 \%$ ) to the CSR series and agrees with IRIS with a standard deviation of 2.8 mas, essentially the same as CSR alone. Thus little has been gained by combining the three series for this period. 
The example shown in Figure $2 c$ illustrates the need for multiple observational programs, and the value of a combined solution when one of the techniques does not provide an adequate result. Clearly care must be taken in the determination of the weights in the combined solution, as shown in the case of the 1984-85 data. In the future, as the observing programs for the two most accurate methods (Satellite Laser Ranging and Radio Interferometry) become well established and routine, the combined solution will probably be derived from these two series alone. However, the astrometric and other observational techniques will continue to be useful in the understanding of systematic differences, and perhaps in the determination of decadelength low frequency variations of the pole which are not possible to study with such short periods of observation.

ACKNOWLEDGEMENT. This research was supported in part by the Crusta1 Dynamics Program of NASA, Grant NAG5-756.

\section{REFERENCES}

Eubanks, T., Steppe, J., Dickey, J., 1985. 'The Atmospheric Excitation of Earth Orientation Changes During Merit', in Proceedings of the Internationa1 Conference on Earth Rotation and the Terrestrial Reference Frame, Volume 2, I. Mueller editor, The Ohio State University, Dept. of Geodetic Science.

Feisse1, M., 1982. 'Combination of Earth Rotation Parameters in 1980 By Various Techniques'. in High Precision Earth Rotation and Earth-Moon Dynamics, O. Calame editor, D. Reidel.

Steppe, J. Eubanks, T., and Spieth, M., 1985. 'Intercomparison of Polar Motion Measurements During the Merit Period', in Proceedings of the International Conference on Earth Rotation and the Terrestrial Reference Frame, Volume 2, I. Mueller editor, The Ohio State University Department of Geodetic Science.

Vicente, R., and Wilson, C., 1986. 'Polar Motion Estimates From Linear Combinations of Independent Series', Astron. Nachr, 307, pp. 137147.

Wilson, C., and Vicente, R., 1986. 'The Combination of Polar Motion Observations', Proceedings of the IAG Symposium, Figure and Dynamics of the Earth, Moon, and Planets, P. Holota, editor, Research Institute of Geodesy, Topography, and Cartography, Prague, Czechoslovakia. 


\section{DISCUSSION}

Schuh: Could you please describe your method of scaling the reported errors?

Reply by Wilson: We multiply the reported errors by a constant so that the mean square value of the modified reported errors agrees with the estimated error obtained by taking differences among independent series.

Vondrak: I came to exactly the same conclusions in a paper that will be published by the end of 1986 in the Bulletin of Astronomical Institutes of Czechoslovakia.

Dickey: You mentioned that astrometric observations could be used for long-term polar motion. However, one should be cautioned that astrometric data has seasonal errors. 